\title{
La toma de decisiones en la atención de la salud mental: el papel del cuidador
}

\author{
Decision making in mental \\ health care: the caregiver's role
}

\begin{abstract}
Kay Wilhelm*
https://doi.org/10.36105/mye.2020v31n4.06

\section{Resumen}

Este artículo establece un marco para considerar los aspectos éticos relacionados con el tratamiento involuntario en personas con: a) una discapacidad intelectual o del desarrollo; b) un impedimento neurológico progresivo, y c) un estado mental comprometido desde el punto de vista del cuidador. Los diferentes momentos de decision de los tres grupos se describen aquí, así como los impactos potenciales en los cuidadores respecto de las decisiones de los pacientes.

Mientras hay algunos aspectos de las capacidades que pueden variar entre estos grupos, otros son transversales y comunes a ellos. Es importante recalcar que todos los cuidadores requieren ser reconocidos y apoyados en las funciones que realizan. Necesitan, por ende, información basada en evidencias y de fuentes confiables sobre las opciones disponibles, para cumplir el rol que
\end{abstract}

\footnotetext{
* Psiquiatra de Enlace, Hospital de San Vicente, Sydney, Australia. Profesor/Jefe de Psiquiatría, Universidad de Notre Dame, Sydney, Australia. Correo electrónico: kay.wilhelm@svha.org.au https://orcid.org/0000-0002-8542-6478 Recepción: 20 de junio de 2020. Aceptación: 15 de julio de 2020.
} 
les toca y ver cómo velar por el propio cuidado de su salud y bienestar para, así, poder cuidar mejor a las personas con las que conviven.

Palabras clave: cuidado de la salud mental, capacidad para tomar decisiones, cuidadores.

Este documento se basa en una plática dada en el reciente Coloquio de la Asociación Internacional de Bioética Católica (AIBC), titulada Promoción de las capacidades en las personas que necesitan apoyo para tomar decisiones sobre la atención de la salud. Tiene como objetivo establecer un marco para examinar las cuestiones éticas relacionadas con el tratamiento involuntario en personas con discapacidades intelectuales y de desarrollo, y con el deterioro cognitivo progresivo o con las enfermedades mentales, desde la perspectiva del cuidador. También tiene como objetivo considerar estas cuestiones éticas y ofrecer algunas soluciones. Se discutirán varios aspectos: ¿Cuál es el contexto para la toma de decisiones? ¿Cuáles son los elementos del cuidado ético desde la perspectiva del cuidador? ¿Qué necesitan los cuidadores?

En este documento sólo se proporcionan principios generales, ya que los países difieren en su marco médico-legal en lo que respecta a la atención involuntaria. También, en este documento, la persona afectada se denomina principalmente «la persona», aunque a veces puede ser un paciente, cliente o consumidor, según el modelo de atención de salud mental aplicada y la etapa del estado de salud mental de la persona.

Mi conclusión será que los cuidadores pueden asociarse con los profesionales de la salud para promover las capacidades de una amplia gama de personas que necesitan apoyo en la toma de decisiones sobre atención a la salud. Sin embargo, el enfoque podría variar, porque las diferentes condiciones de salud tienen diferentes cursos de tiempo e impactos en las personas afectadas y en sus cui- 
sólo de superar la enfermedad, sino también de preservar la salud. Por lo tanto, la prevención y la rehabilitación tienen papeles cada vez más importantes que desempeñar junto con las terapias curativas.

Los cuidadores no están realmente incluidos en el modelo biomédico. Un avance ha sido el modelo biopsicosocial de atención médica, que analiza la salud mental y los sistemas sociales en torno al individuo en términos de cómo éstos impactan al individuo. Este modelo se utiliza más en entornos de salud mental y rehabilitación, y considera al consumidor de dichos servicios como parte de un sistema en equilibrio. Esto implica que hay varias maneras de abordar el problema de la enfermedad y la falta de bienestar. El modelo biopsicosocial también se centra en las fortalezas y en la capacidad, en lugar de simplemente identificar la debilidad y la incapacidad en los pacientes.

El modelo social es un modelo alternativo que se centra más en el acceso a la atención médica y a la colaboración intersectorial entre la salud, el gobierno, las organizaciones no gubernamentales (ONG) y los sectores privados. Este modelo considera las inequidades sociales (como las que se basan en el género, la cultura, la raza, el estado económico o surgen debido a la exclusión social) y los determinantes del comportamiento (según la influencia del género, la cultura, la raza o el origen étnico, el estado socioeconómico, la ubicación y el estado físico). Este modelo busca empoderar a los individuos y a las comunidades, así como las estrategias de promoción de la salud. Al igual que el modelo biopsicosocial, el modelo social considera los sistemas y, aunque no se tienen en cuenta específicamente, a los cuidadores como parte también de los sistemas. Este modelo es más aplicable a las enfermedades crónicas y particularmente relevantes para los cuidadores, que están trabajando en cómo interactuar con los sistemas y abogar por su consumidor individual o grupo de consumidores.

Los modelos anteriores implican que los cuidadores tienen algún agenciamiento. Sin embargo, hay otros modelos (como un modelo de conspiración), en el que los consumidores son vistos simplemente 
como fuera de sintonía con la corriente principal, y en los que se percibe que la sociedad conspira para forzar el tratamiento de las personas vulnerables. Aquí, los cuidadores consideran que la sociedad no tiene derecho a evitar que los individuos "sean ellos mismos», sino que deben adaptarse a la persona en lugar de tratar de cambiarla. En ocasiones, los cuidadores pueden tener su propia experiencia con la afección, el tratamiento y el sistema de salud, lo que puede llevar a que desconfíen de ese sistema.

En el pasado, las familias cuidaban a las personas con discapacidad o problemas de salud mental. Estas personas a menudo estaban escondidas en casa o colocadas en instituciones. Con los avances médicos mencionados anteriormente, las personas con una variedad de discapacidades o enfermedades mentales viven más tiempo: ellos y sus cuidadores tienen una visión menos pesimista de lo que podría hacerse, y aumentan las expectativas con respecto a mejorar su calidad de vida. Junto con esto, se ha dado la desinstitucionalización en las últimas décadas, lo que ha aumentado la carga sobre los cuidadores.

\section{b) Diferentes tipos de cuidadores implican diferentes roles}

La variación entre los cuidadores puede incluir: la cantidad de cuidadores que tiene una persona, la calidad del apoyo, el lugar donde los cuidadores se colocan en el viaje del cuidado en relación con la persona, sus expectativas y nivel de acuerdo, y si parecen estar actuando en el interés de la persona a quien están cuidando.

También hay muchos tipos de cuidadores, incluyendo a las familias nucleares y extendidas, padres solteros, parejas del mismo sexo, padres de crianza o adoptivos, parejas, niños que actúan como únicos agentes, amigos y cuidadores designados. Este rango de posibles grupos de cuidado ha evolucionado en las últimas décadas.

Al mismo tiempo, el énfasis en el papel de estos cuidadores está evolucionando para reflejar las costumbres, culturas, normatividad y políticas judiciales. Por lo tanto, el énfasis en los derechos de los 
cuidadores de enviar a sus seres queridos enfermos al hospital o aceptar el tratamiento puede variar con el tiempo y según la jurisdicción. En términos generales, donde la mayoría de las jurisdicciones declararon previamente que el «pariente más cercano» tenía derecho a apelar y quejarse, ahora a menudo hay disposiciones más formales para que un apoderado designado o sustituto tome decisiones sobre la salud, bienestar y decisiones financieras, si la persona carece de capacidad para tomar tales decisiones en el momento del tratamiento propuesto, y también para que las personas den instrucciones por adelantado. Además, hay un mayor reconocimiento del equilibrio de los derechos de los familiares frente al abuso por parte de los mismos familiares, y muchas jurisdicciones establecen límites y salvaguardas para dar cuenta de ello.

\section{c) Diferentes puntos en el viaje del cuidador}

Mientras que los consumidores de servicios de salud mental están en un viaje personal para lidiar con su condición, también lo hacen sus cuidadores. En la tabla 1 observamos los problemas relacionados con el viaje del cuidador. Si bien ésta es una simplificación de los pasos, es importante reconocer que los cuidadores pasan por diferentes etapas, aunque algunos podrían quedar «atascados» en una etapa en particular.

\section{Condiciones diferentes}

En este coloquio, estamos considerando tres tipos de condiciones que pueden afectar el funcionamiento cognitivo y el proceso de toma de decisiones de la persona:

1. Discapacidades intelectuales y del desarrollo: incluyen trastornos genéticos, malformaciones congénitas, condiciones adquiridas tempranamente en la vida, autismo, dificultades de aprendizaje.

2. Enfermedades neurológicas progresivas: incluyen demencia, daño cerebral y afecciones neurológicas (como la enfermedad de Parkinson y una variedad de lesiones intracerebrales). 
La toma de decisiones en la atención de la salud mental: el papel del cuidador

Cuadro 1. Etapas del viaje de un cuidador.

Se señalan algunas emociones típicas asociadas con éstas.

\begin{tabular}{|c|c|c|}
\hline $\begin{array}{l}\text { Etapas del viaje } \\
\text { del cuidador }\end{array}$ & $\begin{array}{l}\text { Emociones } \\
\text { asociadas }\end{array}$ & $\begin{array}{l}\text { Factores relacionados } \\
\text { con el impacto }\end{array}$ \\
\hline $\begin{array}{l}\text { Darse cuenta de que algo } \\
\text { está mal: puede ser rápido } \\
\text { con un comienzo agudo o } \\
\text { una realización gradual. }\end{array}$ & $\begin{array}{l}\text { Preocupación, } \\
\text { miedo, pánico. }\end{array}$ & Desconcierto. \\
\hline $\begin{array}{l}\text { Haciendo preguntas, } \\
\text { aceptando la situación. }\end{array}$ & $\begin{array}{l}\text { Desconcierto, ansiedad o } \\
\text { confianza creciente, } \\
\text { sentirse valorado o sentirse } \\
\text { abrumado, conflictivo. }\end{array}$ & $\begin{array}{l}\text { Depende de que los } \\
\text { profesionales de la salud } \\
\text { aborden sus preguntas, } \\
\text { desarrollen un plan con } \\
\text { ellos; del nivel de compro- } \\
\text { miso y de la coincidencia } \\
\text { de modelos entre los } \\
\text { profesionales de la salud } \\
\text { y los cuidadores. }\end{array}$ \\
\hline $\begin{array}{l}\text { Ser un representante } \\
\text { del paciente. }\end{array}$ & & $\begin{array}{l}\text { Confianza en conocer } \\
\text { las necesidades y deseos } \\
\text { del paciente. }\end{array}$ \\
\hline $\begin{array}{l}\text { Ajustándose a su papel } \\
\text { y a las implicaciones } \\
\text { para su propia vida. }\end{array}$ & $\begin{array}{l}\text { Ajuste/aceptación o } \\
\text { resentimiento. }\end{array}$ & $\begin{array}{l}\text { Depende de su propia } \\
\text { vida y del apoyo de los } \\
\text { demás. }\end{array}$ \\
\hline $\begin{array}{l}\text { Abogar con los sistemas } \\
\text { médicos y guberna- } \\
\text { mentales. }\end{array}$ & $\begin{array}{l}\text { Desconcierto, ansiedad o } \\
\text { creciendo la confianza, } \\
\text { sintiéndose valorado. } \\
\text { Puede ser una sensación } \\
\text { de alivio, pero también } \\
\text { implica una pérdida de } \\
\text { valor y significado; sentirse } \\
\text { abandonado. }\end{array}$ & $\begin{array}{l}\text { Depende de las cuestiones } \\
\text { que se aborden; de la } \\
\text { elaboración de un plan; } \\
\text { del nivel de compromiso; } \\
\text { de la coincidencia de los } \\
\text { modelos y del grado de } \\
\text { apoyo social. }\end{array}$ \\
\hline $\begin{array}{l}\text { Posiblemente renunciando } \\
\text { al papel de cuidador, o } \\
\text { siendo capaz de dar un } \\
\text { paso atrás en el papel } \\
\text { de cuidador. }\end{array}$ & & $\begin{array}{l}\text { Se supone que los } \\
\text { cuidadores se sentirán } \\
\text { aliviados, pero cuando } \\
\text { los cuidados han ocupado } \\
\text { gran parte de la vida del } \\
\text { cuidador, puede ser un } \\
\text { momento de emociones } \\
\text { contradictorias. }\end{array}$ \\
\hline
\end{tabular}

Fuente: Elaboración propia.

Medicina y Ética - Octubre-Diciembre 2020 - Vol. 31 - Núm. 4 
3. Enfermedades mentales y adicciones: incluyen afecciones agudas (delirio, intoxicación, respuestas a estrés extremo y trauma); trastornos del estado de ánimo (depresión, manía, trastorno bipolar); trastornos psicóticos (principalmente esquizofrenia, trastornos bipolares); trastornos de la alimentación; adicción (nicotina, drogas, juego, medicamentos prescritos o no prescritos, y alcohol).

\section{a) Diferentes tipos de toma de decisiones involuntarias}

Esto no pretende ser una revisión completa de los diversos requisitos legales para la detención y el tratamiento involuntarios y las implicaciones para la toma de decisiones. Puede haber usos tempo-

Cuadro 2. Comparación de los tres tipos de afecciones y sus implicaciones para los cuidadores.

\begin{tabular}{|c|c|c|}
\hline $\begin{array}{l}\text { Etapa de inicio } \\
\text { de la vida }\end{array}$ & Implicaciones & $\begin{array}{l}\text { Impacto en } \\
\text { el cuidador }\end{array}$ \\
\hline \multicolumn{3}{|c|}{ Discapacidades intelectuales y de desarrollo. } \\
\hline $\begin{array}{l}\text { - Presente desde el } \\
\text { nacimiento/niño } \\
\text { (condiciones congénitas o } \\
\text { ambientales, retraso en el } \\
\text { desarrollo, autismo, } \\
\text { resultado de una enferme- } \\
\text { dad de la infancia). } \\
\text { - Puede ser estable, o } \\
\text { fluctuante con declive } \\
\text { funcional. }\end{array}$ & $\begin{array}{l}\text { - Grandes repercusiones } \\
\text { en el desarrollo, la } \\
\text { educación, las habilidades } \\
\text { sociales. } \\
\text { - La respuesta variará con: } \\
\text { - Cantidad de apoyo } \\
\text { disponible (familia/ } \\
\text { amigos/atención } \\
\text { sanitaria/otros } \\
\text { sistemas de apoyo). } \\
\text { - Cultura (vergüenza o } \\
\text { aceptación). } \\
\text { - Nivel de comprensión. } \\
\text { - El sentido del agencia- } \\
\text { miento del cuidador. }\end{array}$ & $\begin{array}{l}\text { - Sentimientos de culpa, } \\
\text { tristeza, lástima, ira (difícil } \\
\text { de poseer). } \\
\text { - Los padres pueden sentir } \\
\text { culpa o sentirse culpables } \\
\text { (y, por lo tanto, pueden } \\
\text { sobrecompensar). } \\
\text { - Los padres son más } \\
\text { propensos a ser los únicos } \\
\text { cuidadores. } \\
\text { - Los cuidadores pueden } \\
\text { tener un sentido de } \\
\text { significado o sentirse } \\
\text { atrapados. } \\
\text { - El apoyo está más } \\
\text { disponible para algunas } \\
\text { condiciones que para otras. } \\
\text { - Los cuidadores y los } \\
\text { médicos pueden sentirse } \\
\text { acostumbrados a posibles } \\
\text { mejoras... }\end{array}$ \\
\hline
\end{tabular}


La toma de decisiones en la atención de la salud mental: el papel del cuidador

\begin{tabular}{|c|c|c|}
\hline \multicolumn{3}{|c|}{ Enfermedades neurológicas progresivas. } \\
\hline $\begin{array}{l}\text { - Surgen en la edad adulta/ } \\
\text { envejecimiento o en } \\
\text { condiciones de fin de vida. } \\
\text { - Problemas de declive } \\
\text { cognitivo continuo, } \\
\text { incluyendo la demencia, } \\
\text { enfrentando la discapacidad. }\end{array}$ & $\begin{array}{l}\text { - La personalidad y los } \\
\text { estilos en la forma de } \\
\text { afrontarlas se han desarro- } \\
\text { llado y darán forma a las } \\
\text { actitudes ante ellas. } \\
\text { - La respuesta de los } \\
\text { cuidadores será más } \\
\text { predecible, pero sigue } \\
\text { estando relacionada con los } \\
\text { mismos problemas que } \\
\text { para las personas con } \\
\text { discapacidades intelectua- } \\
\text { les y de desarrollo. } \\
\text { - Se debe alentar a los } \\
\text { cuidadores a que examinen } \\
\text { las opiniones de la persona } \\
\text { sobre cómo hacer frente a } \\
\text { su disminución progresiva } \\
\text { (incluidas las cuestiones } \\
\text { relativas al final de la vida), } \\
\text { mientras tenga capacidad } \\
\text { para tomar decisiones. }\end{array}$ & $\begin{array}{l}\text { - Sentimientos de ansie- } \\
\text { dad, tristeza, lástima, muy } \\
\text { ligados a la calidad de la } \\
\text { relación. } \\
\text { - Vergüenza con los } \\
\text { comportamientos. } \\
\text { - Enfado, especialmente si } \\
\text { es autoinfligida o se } \\
\text { produce en un momento en } \\
\text { el que el cuidador tenía } \\
\text { planes diferentes (por } \\
\text { ejemplo, viajes, jubilación). } \\
\text { - Los padres pueden tener } \\
\text { un papel, pero la pareja, los } \\
\text { hijos (cuando están presen- } \\
\text { tes) tienen más probabilida- } \\
\text { des de participar. } \\
\text { - Los cuidadores pueden } \\
\text { tener un sentido de } \\
\text { significado o de atrapa- } \\
\text { miento, pero saben que se } \\
\text { trata de un compromiso de } \\
\text { tiempo limitado. }\end{array}$ \\
\hline \multicolumn{3}{|c|}{ La salud mental comprometida y las adicciones. } \\
\hline $\begin{array}{l}\text { - Cuando surgen durante } \\
\text { la vida: } \\
\text { - Cambios de humor: } \\
\text { depresión, manía, } \\
\text { trastorno bipolar. } \\
\text { - Trastornos psicóticos: } \\
\text { psicosis temprana, } \\
\text { esquizofrenia, trastorno } \\
\text { bipolar, psicosis inducida } \\
\text { por drogas. } \\
\text { - Estados agudos: delirio, } \\
\text { intoxicación, respuestas } \\
\text { al estrés extremo y al } \\
\text { trauma. } \\
\text { - Los episodios tienden a } \\
\text { ser episódicos. }\end{array}$ & $\begin{array}{l}\text { - La personalidad y los } \\
\text { estilos en la forma de } \\
\text { afrontarlas se han desarro- } \\
\text { llado y darán forma a las } \\
\text { actitudes ante ellas. } \\
\text { - La respuesta de los } \\
\text { cuidadores será más } \\
\text { predecible, pero aún } \\
\text { relacionada con los mismos } \\
\text { problemas antes indicados. } \\
\text { - La naturaleza temporal } \\
\text { debería permitir la discu- } \\
\text { sión de los problemas y la } \\
\text { planificación anticipada de } \\
\text { los futuros episodios en } \\
\text { recuperación. }\end{array}$ & $\begin{array}{l}\text { - Sentimientos de miedo, } \\
\text { ansiedad, vergüenza, } \\
\text { bochorno, desconcierto. } \\
\text { - Insuficiencia. } \\
\text { - Enojo hacia la persona, } \\
\text { sobre todo si la condición } \\
\text { es vista como autoinfligida. } \\
\text { - Los episodios agudos } \\
\text { pueden ser muy confronta- } \\
\text { dos y atemorizantes para } \\
\text { los cuidadores. } \\
\text { - Los padres pueden tener } \\
\text { un rol, pero la familia } \\
\text { (pareja, hijos) es más } \\
\text { propensa a involucrarse. } \\
\text { - Se prevé que el papel del } \\
\text { cuidador se deteriore tem- } \\
\text { poralmente en salud mental. }\end{array}$ \\
\hline
\end{tabular}

Medicina y Ética - Octubre-Diciembre 2020 - Vol. 31 - Núm. 4 


\begin{tabular}{|c|c|c|}
\hline \multicolumn{3}{|c|}{ Cuando crecen en adolescentes y/o adultos jóvenes. } \\
\hline $\begin{array}{l}\text { - Trastornos de la } \\
\text { alimentación. } \\
\text { - Adicción: nicotina, drogas } \\
\text { (sin receta, alcohol, juego). } \\
\text { - Trastornos de } \\
\text { personalidad. }\end{array}$ & $\begin{array}{l}\text { - Las condiciones que } \\
\text { surjan en este grupo de } \\
\text { edad afectarán a la } \\
\text { personalidad, la madurez, } \\
\text { la red social y la capacidad } \\
\text { de tomar decisiones de la } \\
\text { persona. } \\
\text { - El carácter temporal debe } \\
\text { permitir el examen de los } \\
\text { problemas y la planificación } \\
\text { anticipada de nuevos } \\
\text { episodios de recuperación. }\end{array}$ & $\begin{array}{l}\text { - Es probable que los } \\
\text { padres sigan siendo } \\
\text { importantes como } \\
\text { cuidadores. } \\
\text { - Estos trastornos pueden } \\
\text { hacer que los padres y las } \\
\text { parejas se sientan } \\
\text { indefensos y asustados. }\end{array}$ \\
\hline \multicolumn{3}{|c|}{ Cuando surgen en los adultos mayores. } \\
\hline $\begin{array}{l}\text { - Depresión melancólica } \\
\text { (a menudo debida a una } \\
\text { enfermedad cerebro- } \\
\text { vascular). } \\
\text { - Efectos en el largo plazo } \\
\text { de las enfermedades } \\
\text { mentales crónicas y de } \\
\text { las adicciones, a menudo } \\
\text { asociados con una } \\
\text { mala salud física. }\end{array}$ & $\begin{array}{l}\text { - Las condiciones que } \\
\text { surgen más tarde en la vida } \\
\text { ocurren cuando la persona- } \\
\text { lidad y la capacidad de } \\
\text { decisión de una persona } \\
\text { son más evidentes, y es } \\
\text { más fácil determinar los } \\
\text { deseos de la persona. }\end{array}$ & $\begin{array}{l}\text { - Es más probable que } \\
\text { los cuidadores sean una } \\
\text { pareja, hijos, amigos o } \\
\text { cuidadores pagados. }\end{array}$ \\
\hline
\end{tabular}

Fuente: Elaboración propia.

rales de la toma de decisiones involuntarias, como invocar regulaciones legales para fines de evaluación involuntaria y tratamiento involuntario para afecciones mentales y médicas o tutela temporal. Cualquiera de éstos podría ocurrir si la persona está sufriendo un episodio agudo de enfermedad mental (por ejemplo, esquizofrenia, depresión severa o trastorno bipolar), temporalmente afectada por intoxicación o sobredosis, y en momentos en que la persona tiene deficiencias cognitivas y conductas que desafían a una enfermedad médica. Es probable que los parientes cercanos u otros miembros de la familia y cuidadores designados participen en estas decisiones. La detención y el tratamiento involuntarios pueden ser segui- 
dos por una orden de tratamiento comunitario, que exige el seguimiento después del alta.

Para condiciones duraderas o que empeoran, podría ser necesario contar con una tutela continua, que podría incluir la toma de decisiones para las personas que, según se estima, carecen de capacidad de decisión en esferas como las finanzas, el alojamiento, las intervenciones médicas o psiquiátricas. Por lo general, los miembros de la familia son nominados como tutores, a menos que ellos mismos lo rechacen o se consideren no aptos, inadecuados o no disponibles.

\section{b) Diferentes niveles de apoyo e interés por parte de cuidadores y médicos}

Vale la pena señalar que no todos los cuidadores son útiles y de apoyo para los miembros de la familia que podría tener cierta capacidad para tomar decisiones sobre el cuidado de la salud. ¿Qué pasa si los cuidadores son abusivos o negligentes hacia la persona? ¿Qué sucede si los cuidadores exacerban los problemas de la persona o socavan el tratamiento y el apoyo? ¿Qué sucede si los cuidadores ejercen una «influencia indebida», respondiendo por la persona, hablando sobre ella y/o ignorándola, impidiendo el acceso o el uso de los servicios? ¿Qué pasa si actúan por sus propios intereses, en lugar de por los de la persona afectada? Cuando esto se detecta, generalmente existen mecanismos para lidiar con la situación, siempre y cuando sea sacado a la luz.

Por otro lado, pueden surgir los mismos problemas con los profesionales de la salud y, nuevamente, es necesario abordarlos. Es más probable que esto ocurra en «instituciones totales», sin procesos adecuados de gobernanza externa.

\section{Los elementos de la atención ética desde la perspectiva del cuidador}

Algunos de los principios generales en la toma de decisiones en todos los tipos de afecciones o enfermedades incluyen: a) aun cuando 
no están bien, las personas pueden contribuir a tomar decisiones y dar su consentimiento o consentimiento limitado; b) en su función de apoyo, los cuidadores deben ser claros sobre qué áreas son importantes para la persona afectada; es mejor designar a alguien que pueda hablar por la persona, que comprenda su punto de vista y que parezca tener el interés principal del paciente; c) existen pasos para la toma de decisiones que se pueden utilizar, con algunos problemas generales y otros específicos de la condición, y d) las decisiones deben ser realistas y alcanzables.

Al considerar la mejor manera de apoyar las capacidades de aquellos que necesitan el apoyo de los cuidadores para tomar decisiones de atención médica, también es importante abordar cinco elementos de la atención ética. Éstos se han descrito como la atención, la responsabilidad, la competencia, la capacidad de respuesta y el cuidado de sí mismo (1). Los cuatro primeros ponen en funcionamiento lo que muchos clínicos calibran intuitivamente, mientras que el quinto, que también es importante, puede ser fácilmente pasado por alto por el personal médico y los cuidadores por igual.

Es útil verificar la comprensión, las expectativas, la experiencia y el modelo de atención de los cuidadores con los que operan. Puede haber o no un «ajuste» entre el modelo de atención de médicos y cuidadores, y lo que es más importante, no todos los profesionales de la salud pueden usar el mismo modelo y pueden o no ser conscientes de estas diferencias. Esto puede ocasionar que se entreguen mensajes contradictorios a los cuidadores. Incluso cuando hay otras personas involucradas en el cuidado de una persona, a menudo se recurre a la familia de la persona, porque los miembros de la familia generalmente están cerca cuando nadie más está disponible.

A su vez, los cuidadores pueden proporcionar más fácilmente una atención respetuosa, incluido el apoyo a las capacidades de toma de decisiones, si tienen un papel claro y son respetados por los demás y por ellos mismos. Para hacerlo, necesitan acceso a fuentes 
La toma de decisiones en la atención de la salud mental: el papel del cuidador

confiables de información de médicos, grupos de apoyo, internet disponible e información local.

Sin embargo, los cuidadores son humanos, y los miembros de la familia a menudo tienen respuestas emocionales mixtas (por ejemplo, alivio, culpa, ira, tristeza, preocupación, estrés) cuando tratan con su pariente en situaciones que ocasionan un tratamiento involuntario, especialmente si la situación es duradera y tienen difícil relación con los profesionales de la salud. Esto puede llevar a situaciones de alta emoción. A veces, se complica por el hecho de que las relaciones con los cuidadores se han desgastado o fracturado antes del episodio agudo. Particularmente en el caso de algunas enfermedades mentales, la familia y los amigos han perdido contacto con la persona por una variedad de razones (por ejemplo, no confían en la persona, han sido lastimados por ellos o tienen miedo debido a los pensamientos delirantes que los involucran, han sido la fuente de trauma o han sido vistos como 'sofocantes'). En el caso de los trastornos de largo plazo, pueden desgastarse si no tienen suficiente respiro o apoyo. Sin embargo, cuando la persona afectada ha perdido la participación de miembros de la familia, una admisión involuntaria podría ser una oportunidad para que se vuelvan a conectar con su familia.

Las posibles barreras para la participación del cuidador en el apoyo a la toma de decisiones de atención médica incluyen la falta de apoyo adecuado del cuidador, la falta de recursos del proveedor de servicios de salud y los pacientes que se consideran demasiado peligrosos y/o erráticos para participar en el proceso.

\section{4. ¿Qué necesitan los cuidadores?}

El cuadro 3 tiene una historia desde el punto de vista del cuidador. Ilustra algunas de las dificultades y emociones mixtas asociadas con ser un cuidador en episodios agudos de enfermedad mental. Un estudio australiano reciente (2) entrevistó a 19 miembros de la 
Cuadro 3. La perspectiva de un cuidador.

Johan es un hombre de 35 años (casado durante 3 años, sin hijos), con una maestría, y ha tenido una serie de trabajos de alto perfil. El informe de su familia (abajo) señala que él había estado «emocionalmente arriba y abajo» y había tomado algunas impulsivas decisiones personales y comerciales en el pasado, pero siempre había logrado liberarse, debido a su alta inteligencia y encanto personal. Sin embargo, en los últimos tres meses, ha estado muy irritable y durmiendo muy mal, y fue despedido del trabajo por su rudeza, después de varias advertencias. Su amigo le ha estado dando cannabis y cocaína "para calmarlo», pero se volvió cada vez más errático y agresivo, y ha acusado a su esposa de infidelidad.

Su esposa está desesperada y se acercó a su suegra en busca de ayuda. Eventualmente llamaron al Equipo de Salud Mental Aguda de la comunidad, lo que llevó a la admisión involuntaria en un centro de salud mental bajo la Ley de Salud Mental local. Johan fue diagnosticado con un episodio maniaco como parte del trastorno bipolar. Después del alta, dejó de tomar todos los medicamentos, fumaba cannabis diariamente y se volvió mucho más errático. Su esposa ahora ha dejado el país, y él ha sido desalojado de su hogar. Johan no quiere que su madre «interfiera con su cuidado», pero sí quiere vivir con ella ahora que está en la indigencia. A continuación, el punto de vista de ella.

Nunca me di cuenta de que era una enfermedad. Era brillante, un poco excéntrico, errático, aunque nunca se le llamó así. Hay muchos ejemplos de él metiéndose en un montón de problemas. Vio a un consejero un par de veces... Él es muy inteligente, con titulos en negocios y contabilidad. Nos convenció para comenzar un negocio. Mirando hacia atrás, se encerró y se deprimió y, en otras ocasiones, estaba drogado. El negocio duró de 2003 a 2007. Se siente culpable por perder cientos de miles de dólares; en 2007 el negocio cerró, pero luego se abrió paso en un prestigioso curso de maestría (habló con el decano una semana antes de que comenzara). Después de eso, fue a Asia, donde se desplomó; parecía muy deprimido. Regresó a Sydney, en octubre/noviembre de 2017; comenzó a actuar de manera errática, cortó todo contacto; su esposa, angustiada, vino a pedirme consejo. Llamamos al hospital. Preguntaron: 
La toma de decisiones en la atención de la salud mental: el papel del cuidador

«¿qué quieres hacer?» Nos dieron opciones, pero no sabiamos realmente las consecuencias, y sabiamos que el personal quería que tomáramos decisiones. Me preguntaban: «iEs un peligro para si mismo? ¿Para otros?» Era difícil saber qué hacer, y teníamos miedo de que esto se propagara. Le pedimos al personal médico que viniera a visitarnos. Después de ser llevado al hospital esposado y detenido como paciente involuntario, asistió a la audiencia del $\mathrm{Ma}$ gistrado una semana después. Estaba más asentado en esta etapa, pero lejos de recuperarse. Fue dado de alta, ya que su amigo había respondido por él en el Tribunal. Su esposa estaba preocupada por su comportamiento; la estaba culpando por tenerlo encerrado. Se encontraron en un lugar abierto. Era volátily aterrador. Fue un desastre. Su esposa estaba asustada por su seguridady la suya. Regresé al servicio de salud mental y pregunté: "¿Qué podemos hacer?» Dijeron que me visitarian y me avisarian antes de irse. Nos visitaron, pero no me avisaron hasta más tarde y nadie respondió a su puerta. Esto está haciendo más daño que bien. Más tarde lo encontré en mi jardín en un estado terrible. No babia tomado ningún medicamento durante dias; su casero lo habia echado y se mudó conmigo. Estaba muy deprimido y arrepentido. Se irrita si algo de esto se discute. Fue a otra clínica y obtuvo ayuda útil (psiquiatra y psicólogo), pero todavía está luchando y ha perdido todo. Ha aumentado el consumo de tabaco y sigue fumando THC. Si le cuento a alguien sobre mis preocupaciones, es probable que se entere y se enoje mucho. No puedo volver a pasar por todo el proceso y no quiero ponerlo fuera de juego, ya que no tiene a nadie más. También quiero lo mejor para él y su futuro. Te sientes muy sola, no sabes a quién preguntar. Es una gran responsabilidad vivir con ellos.

familia que tenían a un miembro detenido involuntariamente, principalmente por una enfermedad psicótica. Este suele ser un momento muy estresante para los cuidadores, que buscan respuestas y se adaptan a las realidades de la condición del miembro afectado. Notaron cuatro elementos críticos que ayudaron a las familias a proporcionar apoyo orientado a la recuperación, los cuales incluyen: 
1. Asegurarse de que los cuidadores tengan confianza en que su pariente está seguro y que recibe una buena atención.

2. Informar a las familias del progreso de sus familiares.

3. Permitir el acceso a la información, y

4. Trabajar en conjunto con las familias.

Llegaron a la conclusión de que «el bienestar familiar mejora cuando se sienten parte de un equipo de apoyo», y recomendaron que las familias deberían tener contacto y acceso a la información sobre su pariente, además de que ésta también era importante para los profesionales de la salud, a fin de ureconocer las relaciones de las familias, la experiencia y la comprensión de su miembro de la familia».

Como parte de los elementos de la atención ética, incluido el apoyo a las capacidades de toma de decisiones, los cuidadores también deben tener la oportunidad y el estímulo para el autocuidado de los pacientes y de ellos mismos. Un reciente estudio australiano utilizó el Programa de Salud Óptima, una serie de módulos ofrecidos a pacientes y cuidadores, para abordar las preocupaciones de los cuidadores y mejorar la autoeficacia de los pacientes en crisis y mantener el bienestar, al tiempo que les ayuda a comprender mejor el sistema de salud y su propio trato. Este programa se ha utilizado para personas afectadas por un derrame cerebral y para sus cuidadores (3). Intervenciones similares destinadas a promover el bienestar del cuidador han sido importantes para apoyar a familias con un miembro con demencia. Son menos comunes para familias con un miembro con enfermedad mental.

En general, los cuidadores buscan y deben participar en la toma de decisiones compartidas con profesionales de la salud, para apoyar a la persona a la que ambos brindan atención. El trabajo de Elwyn et al., proporciona un modelo de 3 pasos para la toma de decisiones compartidas: 1) introduce las opciones; 2) describe las opciones, a menudo integrando el uso de los apoyos de decisión del paciente, y 3) ayuda a los pacientes a explorar preferencias y a 
tomar decisiones. Esto implica un proceso en el que tanto los cuidadores como los médicos trabajan con la persona para tomar decisiones basadas en «lo que más le importa a la persona» (4). Este proceso también se comparte en el libro de Gawande sobre cómo abordar los problemas relacionados con el final de la vida útil (5).

Algunos otros modelos para mejorar el apoyo de los cuidadores a los miembros de la familia que vale la pena destacar incluyen la práctica de recuperación centrada en la familia, que se utiliza para apoyar a la familia con el fin de ayudar mejor en la recuperación al familiar que tiene problemas de salud mental y, en segundo lugar, para abordar las necesidades de los propios miembros de la familia (6). Se necesita un marco de recuperación enfocado en la familia para ayudar a los planificadores de servicios, a los profesionales, a los miembros de la familia y a aquéllos con necesidades de salud mental, y dicho marco de recuperación debe estar integrado en las pautas de la práctica. También ha sido exitoso enseñar a las familias a usar un enfoque de resolución de problemas (Terapia de Resolución de Problemas Familiares) para mejorar la función familiar, particularmente en el manejo de situaciones de crisis (7).

Éstas son algunas áreas sugeridas donde la información confiable beneficiaría a los cuidadores, al apoyar a los miembros de la familia con problemas de salud mental:

1. Condiciones específicas de relevancia para ellos (incluido el delirio).

2. Con quién están tratando y cómo sacar el máximo provecho de las interacciones (por ejemplo, cómo aprovechar al máximo una conferencia de caso, una ronda de sala y/o una entrevista con un nuevo personal clínico).

3. Cómo lidiar con crisis y recaídas.

4. Cómo trabajar en equipo y comprometer la solución de problemas familiares.

5. Cómo ser un defensor.

6. Cómo ser un apoderado o sustituto de la toma de decisiones, y en el uso de una «lista de verificación de interés superion». ${ }^{1}$ 
7. Acceso a grupos de apoyo sobre las condiciones de relevancia.

8. Información sobre el autocuidado y el descanso.

Es de destacar que el manual iSupport (8) de la Organización Mundial de la Salud (OMS) para cuidadores de personas con demencia aborda muchas áreas mencionadas anteriormente. Incluye cinco módulos, a saber: a) introducción a la demencia; b) ser un cuidador; c) cuidarme como cuidador; d) proporcionar atención diaria, y e) tratar con cambios de comportamiento.

No existe un manual correspondiente para las personas que cuidan a alguien con problemas de salud mental pero, debido a la gran diversidad y variedad en la gravedad de las condiciones de salud mental, desarrollar dicho manual podría no ser tan simple. Hay organizaciones que brindan apoyo a los cuidadores de personas con trastornos específicos, como psicosis, trastornos alimentarios y depresión. Éstos son demasiado numerosos y diversos para especificar aquí, pero los consejos contenidos en dichos manuales a menudo no son suficientes para los cuidadores que se enfrentan a situaciones agudas y emocionalmente cargadas, como el ejemplo citado en el Cuadro 3.

\section{Conclusión}

Algunas culturas y sociedades han tratado de cuidar a las familias extensas. En otras culturas y sociedades, la atención institucional aún se prefiere para personas con discapacidades intelectuales y del desarrollo, trastornos neurológicos progresivos, salud mental comprometida y adicción. Sin embargo, ahora hay menos acceso a instituciones y familias más pequeñas y diversas. $\mathrm{Al}$ mismo tiempo, ha habido un mayor interés en la transparencia en la atención médica y en el acceso a la información. Estos cambios se han centrado más en un número menor de cuidadores, con el resultado de que el 
La toma de decisiones en la atención de la salud mental: el papel del cuidador

papel del cuidador, junto con las definiciones y las implicaciones médico-legales, ha ido evolucionando.

Los tres grupos de condiciones mencionados tienen diferentes cursos de tiempo e impactos en la persona involucrada y en sus cuidadores. Los enfoques para los cuidadores sobre las capacidades de cuidado y apoyo de sus seres queridos para tomar decisiones de atención médica, por lo tanto, pueden variar entre estas condiciones. Sin embargo, algunos principios se aplican en todos los grupos: los cuidadores necesitan reconocimiento y apoyo para los roles que asumen y, además, acceder a información sobre cómo cumplir mejor estos roles. Deben cuidar su propia salud y bienestar, así como el de la persona a la que cuidan.

\section{Notas bibliográficas}

1 https://www.mind.org.uk/information-support/legal-rights/mental-capacity-act2005/best-interests/\#.XS3atOgzbcs

https://www.google.com/search?q=best+interest+checklist\&rlz=1C1DLBX_en AU835AU835\&oq=Best+interst+checklis

https://www.scie.org.uk/dementia/supporting-people-with-dementia/decisions/bestinterest.asp

\section{Referencias bibliográficas}

1. Verkerk M. La perspectiva del cuidado y la autonomía. Medicina, cuidado de la salud y filosofía. 2001; 4(3): 289-294.

2. Wyder M, Bland Robert, McCann Karen, Crompton David. La experiencia familiar de la crisis del tratamiento involuntario en salud mental. Trabajo Social Australiano. 2018; 71(3): 319-331.

3. Brasier C, Ski C, Thompson D, Cameron J, et al. El Programa de Salud Óptima de Stroke and Carer (PSOSC) para mejorar la salud psicosocial: protocolo de estudio para un ensayo controlado aleatorio. Juicios. 2016; 17(1): 446-455. https://doi. org/10.32597/dmin/512

4. Elwyn G, Frosch D, Thomson R, et al. Toma de decisiones compartida: un modelo para la práctica clínica. Revista de Medicina Interna General. 2012; 27(10): 1361-67. https://doi.org/10.1016/j.rce.2017.06.002

Medicina y Ética - Octubre-Diciembre 2020 - Vol. 31 - Núm. 4 
La toma de decisiones en la atención de la salud mental: el papel del cuidador

dadores. Para todas las condiciones, los cuidadores necesitan reconocimiento y apoyo para las funciones que desempeñan, y acceso a la información sobre la mejor manera de cumplir esas funciones. Deben velar por su propia salud y bienestar, así como por la de la persona que cuidan.

\section{1. ¿Cuál es el contexto para la adopción de decisiones?}

\section{a) Diferentes modelos de enfermedad}

Durante el siglo pasado, muchos países han utilizado un modelo biomédico de atención médica, que se centra en los aspectos biológicos de la enfermedad y padecimiento, e implica el diagnóstico y tratamiento de enfermedades o afecciones una vez que los síntomas están presentes. Este modelo se ha centrado en médicos, profesionales de la salud y hospitales que administran el tratamiento. Aquí el objetivo es tratar a las personas y su condición, con la expectativa de que volverán a un estado de salud previo a la enfermedad. La intervención se centra en los determinantes biológicos de la salud, y menos en los determinantes conductuales y sociales. Este enfoque de intervención médica ha sido asistido por importantes avances en los tratamientos descubiertos durante este tiempo (por ejemplo, medicamentos, técnicas médicas y quirúrgicas y otras formas de tecnología médica), y ha aumentado la presión sobre los hospitales y los profesionales de la salud para diagnosticar con precisión y encontrar curas u otros tratamientos. También han jugado un papel importante en la prolongación de la esperanza de vida, y han aumentado las expectativas del público sobre lo que las intervenciones médicas pueden lograr. El modelo biomédico ha tenido mucho éxito para tratar afecciones agudas, pero necesita «ajustes» cuando se consideran afecciones de por vida o enfermedades crónicas. La medicina contemporánea se enfrenta cada vez más a la tarea, no 
5. Gawande A. Ser mortal: la medicina y lo que importa al final. Nueva York: Metropolitan Books; Henry Holt \& Company; 2014.

6. Ward B, Reupert A, McCormick F, Waller S, Kidd S. Práctica centrada en la familia dentro de un marco de recuperación: perspectivas cualitativas de los profesionales. BMC Health Serv Res. 2017; 17(1): 234.

7. Epstein N, Bishop D, Ryan C, Miller I, Keitner G. El Modelo de McMaster Vista del Funcionamiento Saludable de la Familia. En Walsh A. (Ed.). Nueva York y Londres: Guilford Press. Procesos familiares Normales. 1993; 138-160).

8. Organización Mundial de la Salud. Apoyo a la demencia. Manual de capacitación y apoyo para cuidadores de personas con demencia. https://www.who.int/ mental_health/neurology/dementia/isupport_manual/en/ https://doi.org/10.17345/ 9788484247661 